\title{
Éducation et autonomie régionale en Espagne
}

Juan Carlos González Faraco et Antonio Luzón Trujillo

Traducteur : Valérie Téhio et Pierre-Louis Gauthier

\section{(2) OpenEdition}

\section{Journals}

Édition électronique

URL : http://journals.openedition.org/ries/727

DOI : $10.4000 /$ ries. 727

ISSN : 2261-4265

Éditeur

Centre international d'études pédagogiques

Édition imprimée

Date de publication : 1 septembre 2009

Pagination : 141-148

ISSN : 1254-4590

Référence électronique

Juan Carlos González Faraco et Antonio Luzón Trujillo, « Éducation et autonomie régionale en

Espagne », Revue internationale d'éducation de Sèvres [En ligne], 51 | septembre 2009, mis en ligne le 02 novembre 2019, consulté le 14 novembre 2019. URL : http://journals.openedition.org/ries/727 ; DOI : $10.4000 /$ ries. 727

Ce document a été généré automatiquement le 14 novembre 2019.

(c) Tous droits réservés 


\title{
Éducation et autonomie régionale en Espagne
}

\author{
Juan Carlos González Faraco et Antonio Luzón Trujillo \\ Traduction : Valérie Téhio et Pierre-Louis Gauthier
}

\section{La décentralisation politique : construire un « État des Autonomies »}

1 Lors des nombreuses manifestations qui parcouraient les rues des villes espagnoles pendant les dernières années du régime franquiste, il était courant de crier en chœur, à plusieurs reprises, cette litanie revendicative: «Liberté! Amnistie! Statut d'autonomie !» Ce qui signifiait : récupération des droits et libertés civiques, libération des prisonniers politiques et remplacement d'un État fortement centralisé par un État décentralisé. La décentralisation est, à partir de là, devenue un élément constitutif du projet démocratique pour l'Espagne qu'on s'imaginait en ces années d'incertitude de la longue période de dictature (1939-1975). Le processus complexe et délicat de transition politique qui suivit immédiatement la mort du général Franco se cristallisa juridiquement dans la Constitution de 1978, encore en vigueur. Celle-ci formalisait un modèle nouveau et singulier d'organisation territoriale et politique de l'Espagne appelé "État des Autonomies", modèle intermédiaire entre la pure décentralisation administrative régionale et le modèle fédéral classique. Trente ans après, et au vu de son développement ultérieur, ce modèle peut être qualifié de « quasi fédéral ».

2 Ce processus singulier s'appuyait sur quelques précédents historiques. Depuis le XIX siècle, le régionalisme et le nationalisme, dont la nature et les formulations étaient très différentes selon les régions du pays, faisaient partie du paysage politique espagnol. Pour sa part, le fédéralisme avait été, depuis ses origines, un principe idéologique de la tradition républicaine espagnole. De fait, pendant la brève étape de la Seconde république (1931-1939), un processus d'autonomie régionale s'était développé autour des identités communautaires fondées sur une langue propre (en Catalogne et au Pays basque). La Constitution républicaine de 1931 soutenait que la culture et l'éducation 
dépendaient de l'État, ce qui n'empêchait ni la reconnaissance de l'enseignement de la langue maternelle pour les communautés bilingues, ni l'attribution à la Catalogne et au Pays basque, dans leurs statuts d'autonomie respectifs ${ }^{1}$, du pouvoir de créer et de gérer leurs propres établissements scolaires, dans le cadre constitutionnel de la république.

Le nouvel État autoritaire né de la guerre civile (1936-1939) anéantit totalement ces prémisses de décentralisation, en restaurant un modèle d'État profondément centralisateur, caractéristique, par ailleurs, de l'histoire de la monarchie espagnole des Bourbons. À partir de là, dictature et centralisme devinrent les faces inséparables d'une même monnaie. D'une certaine manière, la Constitution de 1978 s'efforçait de reprendre, à plusieurs années de distance et dans un contexte historique bien différent, le processus de décentralisation républicaine initial, afin de fonder un nouvel État de droit dans lequel soient effectivement reconnues les différences régionales, en tant qu'élément indissociable des droits et libertés démocratiques. Il s'agissait en principe d'une nouvelle tentative pour trouver une solution politique non fédérale au désir de gouvernement autonome des dénommées «communautés historiques» (Catalogne, Pays basque, Galice), sans pour autant provoquer des inégalités par rapport aux autres communautés régionales : un équilibre difficile à tenir. De fait, la constitution établit de claires distinctions entre ces communautés autonomes et les autres (appelées respectivement "nationalités historiques " et "régions»), en proposant des voies juridiques séparées pour réaliser leur autonomie politique et, dans certains cas, en établissant des règles fiscales spéciales, voire très favorables (au Pays basque et en Navarre, par exemple).

4 Avec le temps, le modèle d'autonomie s'est formellement généralisé jusqu'à former une carte asymétrique de 17 Communautés autonomes, dont le gouvernement central doit atténuer et corriger les prévisibles déséquilibres, par tout un jeu d'institutions, de stratégies et de mesures de coopération, de coordination et de redistribution interterritoriales. Malgré cela, les accords bilatéraux entre le gouvernement central et les gouvernements autonomes ont prévalu au fil des années, aux dépens de la solidarité et de la collaboration interrégionales et multilatérales.

Le système autonome a profondément modifié l'organisation politique traditionnelle de l'État et a sans doute amélioré son efficacité administrative, mais il a aussi démontré des faiblesses fondamentales. On peut dire que l'Espagne est aujourd'hui un "État polycentriste " plutôt qu'un État décentralisé totalement et à tous les niveaux. Les institutions politiques autonomes s'efforcent, dans un mouvement de revendications sans fin, d'obtenir que l'État leur cède toujours plus de ressources économiques et de compétences politique mais elles tendent aussi à défendre leur totale ou presque totale capacité de décision face à des instances inférieures, de dimension provinciale, régionale ou locale. Dans le champ de l'éducation, comme nous allons le voir, l'État a transféré aux gouvernements autonomes l'essentiel de ses compétences ${ }^{2}$. En conséquence, la capacité de décision des municipalités et autres instances locales est très faible et sans effet, si on la compare à celle des gouvernements régionaux.

\section{Le processus de décentralisation éducative}

$6 \quad$ Il y a en Espagne un seul système éducatif national dont l'organisation générale relève de l'État, mais dont la régulation spécifique, la gestion et le financement effectifs dépendent fondamentalement des gouvernements autonomes. L'État, comme dans les 
autres domaines de la politique nationale, se réserve les compétences générales afin de garantir de l'égalité de tous les Espagnols dans l'exercice de leurs droits citoyens fondamentaux. Dans le champ de l'éducation, l'État a le devoir de garantir l'accès de tous à l'éducation de façon égale et sur tout le territoire national, cependant que les Communautés autonomes ont pour fonction de développer, de contrôler et d'exécuter la politique éducative sur leurs territoires respectifs, dans le cadre de la constitution.

7 Le processus décentralisateur, toujours inachevé et discuté, s'est développé lentement, avec difficulté et de manière graduelle au cours des trente dernières années. La transition d'un État profondément centralisé vers un État à caractère quasi-fédéral (mais dont la décentralisation est plus forte que dans certains pays traditionnellement fédéraux) a été complexe et ambivalente, sans doute faute de tradition décentralisatrice dans l'histoire espagnole. Ce processus s'est développé en trois phases successives clairement distinctes. La définition d'une nouvelle carte de l'autonomie a commencé pendant la transition politique, avec la promulgation des statuts d'autonomie de la Catalogne et du Pays basque. Par la suite, on a établi un pacte politique pour étendre le processus d'autonomie à d'autres Communautés ayant des particularités historico-géographiques et culturelles (Andalousie, Galice, Navarre, Communauté de Valence et des Canaries). Enfin, en 2000, le processus a culminé avec le transfert de compétences de l'État vers le reste des Communautés, en fonction de leurs statuts respectifs. Dans le domaine de l'éducation, la même évolution progressive a été suivie. Initialement, l'administration de l'éducation revenait au ministère de l'Éducation et de la science sur la majeure partie du territoire national, à l'exception de la Catalogne et du Pays basque. Peu à peu, le ministère a cédé des compétences aux autres Communautés autonomes, immédiatement pour certaines, comme l'Andalousie, ultérieurement pour d'autres. À partir de 2000, le transfert de compétences en matière éducative a été complètement finalisé. Cela a impliqué un effort constant et important de coordination afin d'éviter une différenciation et une hétérogénéité de situations telles qu'elles eussent provoqué des déséquilibres, des inégalités et des injustices comparables. Cet effort n'a pas toujours atteint ses objectifs, et a provoqué toute une série de conflits.

\section{Le pouvoir en éducation, entre gouvernement central et Communautés autonomes}

8 Actuellement, quelles sont les compétences éducatives qui reviennent à l'État, c'est-àdire au gouvernement central et quelles sont celles qui dépendent des Communautés autonomes, c'est-à-dire des gouvernements autonomes ou régionaux ?

9 Les compétences éducatives relevant de l'État sont réunies dans l'article 149 de la Constitution, qui définit l'État comme le garant du droit à l'éducation, et par conséquent, comme le principal responsable de l'ordonnancement et de la régulation générale du système éducatif ainsi que de l'impulsion donnée en faveur de politiques de compensation et de redistribution des ressources. Il revient donc à l'État d'établir, au moyen de lois générales, les niveaux scolaires, les cycles et modalités d'enseignement, la politique d'aides et de bourses, et la liste des enseignements fondamentaux ou communs qui font partie du curriculum national de base. Ces enseignements fondamentaux seront cependant développés par les Communautés autonomes en fonction des compétences que leur attribuent leurs statuts respectifs, avec des 
différences qui, malgré la variété des statuts, restent peu marquées dans le champ de l'éducation.

La principale différence observable concerne les communautés qui ont une langue officielle propre (Catalogne, Pays basque, Galice, Navarre, Communauté de Valence et des Îles Baléares). Alors que, dans les autres communautés, l'État définit $65 \%$ du curriculum, l'État n'en détermine que $55 \%$ dans ces communautés. La différence est logiquement due à la nécessité d'inclure l'enseignement des deux langues officielles, ce qui pose d'innombrables problèmes et provoque des polémiques dans les communautés régies par des gouvernements nationalistes. Dans ces cas-là, il est fréquent que soit privilégiée l'immersion linguistique dans la langue propre, au détriment du castillan, ce qui peut faire obstacle à cet enseignement, le mettre en péril ou conduire à ne pas respecter les programmes nationaux communs, notamment pour l'enseignement des sciences sociales (géographie, histoire, etc.).

Compétences éducatives de l'État et des communautés autonomes

\begin{tabular}{|c|c|}
\hline Relevant de l'État & Relevant des Communautés autonomes \\
\hline $\begin{array}{l}\text { Régulation des conditions qui garantissent l'éga- } \\
\text { lité des citoyens dans l'exercice de leur droit à } \\
\text { l'éducation. }\end{array}$ & $\begin{array}{l}\text { Mise en œuvre de la réglementation approuvée } \\
\text { par l'État. }\end{array}$ \\
\hline $\begin{array}{l}\text { Régulation des conditions d'obtention, de déli- } \\
\text { vrance et d'homologation des diplômes. }\end{array}$ & $\begin{array}{l}\text { Gestion directe du secteur éducatif en tant que ser- } \\
\text { vice public. }\end{array}$ \\
\hline $\begin{array}{l}\text { Approbation des normes fondamentales pour le } \\
\text { développement des principes de base de la poli- } \\
\text { tique éducative (article } 27 \text { de la Constitution). }\end{array}$ & $\begin{array}{l}\text { Certification de tous les établissements publics à } \\
\text { tous les niveaux d'enseignement. Construction et } \\
\text { aménagement des Etablissements scolaires et mise } \\
\text { en place d'accords éducatifs avec des entités pri- } \\
\text { vées et d'autres ressources. }\end{array}$ \\
\hline $\begin{array}{l}\text { Gestion de l'éducation des villes africaines auto- } \\
\text { nomes de Ceuta et Melilla. }\end{array}$ & $\begin{array}{l}\text { La gestion des enseignants et l'inspection des éta- } \\
\text { blissements. d'enseignement. }\end{array}$ \\
\hline $\begin{array}{l}\text { Gestion des établissements scolaires à l'étranger et } \\
\text { du ministère de la Défense. }\end{array}$ & $\begin{array}{l}\text { La supervision, le contrôle et la coordination de } \\
\text { l'offre scolaire et des postes. }\end{array}$ \\
\hline Politique de bourses et d'aides aux études. & $\begin{array}{l}\text { Approbation du curriculum pour les différents } \\
\text { niveaux communs d'enseignement fondamental } \\
\text { fixés par l'État. }\end{array}$ \\
\hline Définition des enseignements fondamentaux. & $\begin{array}{l}\text { Élaboration, approbation et exécution des pro- } \\
\text { grammes d'investissements. }\end{array}$ \\
\hline $\begin{array}{l}\text { Contrôle de l'application des lois nationales par } \\
\text { les Communautés autonomes. }\end{array}$ & Gestion des bourses et aides approuvées par l'État. \\
\hline
\end{tabular}

Source : Bonal, Rambla, Calderón et Pros, 2005, pp. 35 et 38.

Pour une plus grande clarté, le tableau suivant réunit, sous forme comparée, les compétences éducatives de l'État et des Communautés autonomes.

Le développement de ce cadre légal, en apparence très homogène, a cependant produit des résultats manifestement inégaux dans chaque Communauté, du fait des différences économiques et socioculturelles structurelles entre les différentes Communautés et également du fait de leur capacité différente à assumer la construction de leur avenir. Ainsi, à titre d'exemple, en comparant les taux d'analphabétisme entre 1887 et 1981, on peut distinguer une « Espagne analphabète » (Andalousie, Canaries, Castille-La Manche, Estrémadure et Murcie) et une "Espagne alphabétisée " (Navarre, Pays basque, Catalogne, Madrid...). L'évolution de la dépense par élève dans les différentes 
Communautés (bien plus importante dans le Pays basque et en Navarre qu'en Estrémadure, Andalousie, et en Castille-La Manche) est un autre exemple éloquent.

Dans le but de résorber ou au moins d'atténuer ces inégalités et asynchronies en termes de croissance et de développement interrégional, on a créé des organismes nationaux de coordination et d'articulation éducative entre les Communautés autonomes et le ministère de l'Éducation.

Ainsi, en 1985, a été créée la Conférence de l'éducation qui réunit des conseillers ou responsables de l'éducation de toutes les Communautés autonomes et du ministère de l'Éducation. Pour beaucoup, cette institution n'a jusqu'à présent pas joué un rôle pertinent en tant qu'instance de coopération interterritoriale, comme cela se passe dans les pays fédéraux. Dans l'intention d'harmoniser et de renforcer la cohérence territoriale, on a aussi créé en 1985 le Conseil de l'école de l'État (chaque Communauté a son propre Conseil de l'école, de même que tout établissement scolaire), en tant qu'organisme national réunissant tous les secteurs impliqués dans la programmation générale de l'enseignement. Il s'agit d'un organisme consultatif, dont la mission est de conseiller le gouvernement en ce qui concerne les lois et projets liés à l'éducation, et de proposer des améliorations du système. Au Conseil de l'école sont représentés tous les secteurs du monde éducatif (enseignants, parents, propriétaires des établissements privés, syndicats, élèves, etc.), ainsi que des personnalités de grand renom.

En 1990, à la base de la première grande réforme éducative engagée par les gouvernements socialistes, est créé l'Institut national de la qualité et de l'évaluation, aujourd'hui appelé Institut de l'évaluation. Il s'agit d'une institution de coopération entre les différentes administrations éducatives, qui est coordonnée par le ministère de l'Éducation, pour le suivi et l'évaluation du système éducatif à tous les niveaux d'enseignement, l'élaboration d'un système d'indicateurs communs et la collaboration dans les études et évaluations internationales (par exemple, l'enquête PISA de l'OCDE). L'existence de cet organisme national n'a pas empêché que certaines communautés aient créé leurs propres mécanismes d'évaluation et de diagnostic, ce qui a provoqué de multiples conflits de compétences entre l'État et les Communautés autonomes, notamment dans le cas de disputes idéologiques ou partisanes.

Comme nous l'avons signalé plus haut, le processus décentralisateur ainsi décrit a généré un pluricentrisme au niveau des décisions politiques en éducation. L'Espagne est passée d'un centre unique, basé à Madrid auprès du gouvernement central, à dixsept centres correspondant chacun à une Communauté autonome. Les gouvernements autonomes, que ce soit par souci de renforcer un processus nationaliste identitaire, ou par exercice jaloux de compétences récemment obtenues, agissent de façon centraliste et assez bureaucratique, sans s'appuyer sur le processus de décentralisation pour le faire évoluer vers des niveaux de décision plus périphériques et par conséquent plus proches des citoyens.

\section{Différences, asymétries et inégalités territoriales}

17 Si nous examinons la carte espagnole de l'éducation et ce, malgré les avancées notables réalisées par les politiques d'équité qui ont été développées à partir des années quatrevingts, on peut se demander si l'effort réformateur a bien conduit aux résultats escomptés. La question est surtout de savoir dans quelle mesure le modèle d'autonomie que nous avons décrit sommairement a contribué à compenser les différences entre les 
Communautés autonomes qui composent l'Espagne. Différents auteurs pensent que la réponse à ces deux questions est négative. En effet, de nombreux indicateurs montrent qu'actuellement, la réalité éducative est plurielle, peu uniforme et faiblement égalitaire: «l'État espagnol comme unité politique et territoriale, à l'intérieur de laquelle se développent des liens solidaires, démontre des inégalités notables quant aux opportunités de scolarisation, en lien avec d'autres inégalités historiques et culturelles dont les racines sont profondes. Il en découle que les citoyens espagnols, malgré une scolarité de base égale pour tous, ne bénéficient pas de probabilités de réussite égales selon l'endroit où ils vivent ni de possibilités de compter avec une éducation préscolaire ou supérieure. Les différentes Communautés autonomes jouissent de ressources et de niveaux de dépense inégaux, de coûts de scolarisation différents, de quotas de scolarisation non-obligatoire différents, d'espérances de scolarité inégales pour leur population, d'offre d'enseignement public différente, et elles ont suivi des rythmes distincts d'implantation des réformes éducatives, etc. » (Gimeno Sacristán, 2005, 39).

Dépense publique par élève non universitaire et par année (2005)

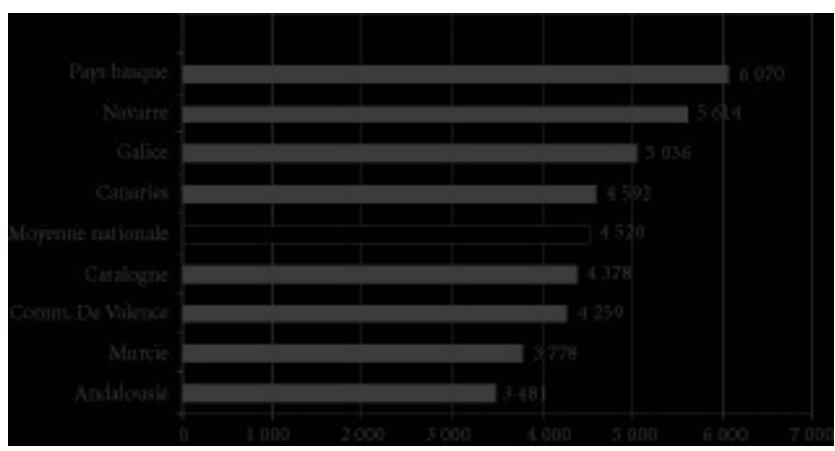

$\mathrm{Si}$, par exemple, nous comparons les résultats du rapport PISA tout au long des dix dernières années, les indices d'abandon scolaire de l'éducation secondaire obligatoire ou inférieure, et la dépense publique par élève non universitaire (figure), nous observons un net désavantage dans le sud de la péninsule et dans les îles (Andalousie, Castille-La Manche, Estré-madure, Canaries), par rapport aux Communautés du nord de la péninsule. Les étudiants du Pays basque, par exemple, profitent d'un niveau élevé de dépense en éducation, du plus bas taux d'abandon des études secondaires postéducation obligatoire (baccalauréat) et obtiennent de bons résultats dans les évaluations internationales. Une autre donnée significative est la différence entre la distribution régionale des secteurs publics et privés. En Andalousie, l'école privée accueille moins de $25 \%$ de la population scolaire, tandis que dans le Pays basque, elle accueille près de $60 \%$. Il est logique de se demander si le modèle autonomiste a renforcé les différences entre les régions ou si, tout au moins, il ne les a pas suffisamment corrigées voire, pis encore, si une fracture Nord-Sud de l'éducation espagnole n'est pas en train de se consolider.

On peut également se demander si le processus décentralisateur a réellement contribué de façon significative au développement éducatif des Communautés autonomes. La réponse n'est pas aisée. Pour commencer, chaque Communauté partait d'une situation distincte. D'autre part, tous les indicateurs ne suivent pas la même logique. Certains dépendent de la richesse de chaque région, d'autres des politiques éducatives développées par les gouvernements autonomes. Le fait est que les systèmes éducatifs 
autonomes n'ont pas réussi, au moins jusqu'à présent, à amortir les disparités territoriales qui, pour la plupart, viennent de loin.

Il est fondamental, pour affronter ce problème, de dépasser la défiance entre les communautés et de développer des actions qui renforcent la solidarité et la coopération les territoires. En ce sens, le rôle de l'État est central, comme garant de l'égalité éducative de tous les citoyens, indépendamment du lieu où ils résident. Les Communautés autonomes gèrent déjà $95 \%$ de la dépense publique en éducation, ce qui démontre que nous nous trouvons face à un système scolaire extraordinairement décentralisé. Cependant, l'équité, la qualité et l'efficacité du système ne peuvent reposer sur la seule décentralisation, mais aussi, et principalement, sur la coordination politique des Communautés autonomes, afin de diminuer le bilatéralisme qui a malheureusement prédominé pendant des années, alors que se mettait en place l'État des Autonomies. Si tel n'était pas le cas, le système éducatif pourrait se décomposer en sous-systèmes disjoints, ce qui causerait un grave préjudice à la cohésion et à l'égalité sociales.

\section{BIBLIOGRAPHIE}

BONAL X., RAMBLA X., CALDERÓN E. et PROS N. (2005) : La descentralización educativa en España. Barcelona, Fundación Pi i Suñer.

GIMENO SACRISTÁN J. (2005) : « Los sentidos y desafíos de la igualdad justa en educación », in Puelles, M. de (coord.), Educación, igualdad y diversidad cultural. Madrid, Biblioteca Nueva.

LUENGO J., SEVILLA D. et TORRES M. (2005) : « From Centralism to Decentralization : The Recent Transformation of the Spanish Education System ». European Education, 37 (1), 46-61.

PUELLES M. de (2004) : « La descentralización de la educación en España : presente, pasado y futuro », in Informe Educativo 2004. Análisis y situación de las Comunidades Autónomas. Madrid, Fundación Hogar del Empleado-Santillana.

\section{NOTES}

1. Le «Statut d'autonomie » est la loi fondamentale de chaque Communauté autonome, une sorte de «constitution» propre, qui définit son modèle de gouvernement autonome et ses compétences politiques fondamentales, dans le cadre de la constitution espagnole. Actuellement, chaque Statut doit être approuvé par le parlement régional puis par le parlement national à une large majorité. Dans quelques Communautés, un référendum populaire est nécessaire.

2. En 1985, les Communautés autonomes contrôlaient seulement $43 \%$ des dépenses totales en éducation; aujourd'hui, elles en contrôlent $95 \%$. 
INDEX

Index géographique : Espagne

Mots-clés : décentralisation, réforme, système éducatif, autonomie, éducation

\section{AUTEURS}

\section{JUAN CARLOS GONZÁLEZ FARACO}

Juan Carlos González Faraco est professeur au sein du département de l'éducation de l'Université de Huelva (Espagne) et professeur adjoint au département d'anthropologie de l'Université d'Alabama (États-Unis). Ses domaines de spécialisation sont l'anthropologie de l'éducation, la formation des enseignants et, plus généralement, les études culturelles et politiques en éducation.

\section{ANTONIO LUZÓN TRUJILLO}

Antonio Luzon Trujillo est professeur d'éducation comparée à la faculté des sciences de l'éducation de l'Université de Grenade (Espagne) et membre du groupe de recherche « Politiques et réformes éducatives » qui dépend du gouvernement autonome d'Andalousie. Ses centres d'intérêt scientifique sont la politique éducative européenne, l'analyse des inégalités éducatives et l'exclusion sociale. Il participe à des projets de coopération universitaire et scientifique avec le Mexique et le Chili. 